\title{
Perception du changement climatique des éleveurs et stratégies d'adaptation aux contraintes environnementales : cas de la commune de Filingué au Niger
}

\author{
Harouna Abdou ${ }^{1 *}$ Ibrahim Adamou Karimou ${ }^{2}$ \\ Boureima Karimou Harouna ${ }^{1}$ Moussa Tahirou Zataou ${ }^{1}$
}

\begin{abstract}
Mots-clés
Eleveur, adaptation aux changements climatiques, enquêtes auprès des ménages, vulnérabilité, résilience, Sahel, Niger
\end{abstract}

Submitted: 13 December 2018

Accepted: 13 April 2020

Published: 29 June 2020

DOI: $10.19182 /$ remvt.31873

\begin{abstract}
Résumé
L'objectif de l'étude était de comprendre la vulnérabilité des élevages de la commune urbaine de Filingué au Niger face aux changements environnementaux. Afin de déterminer, d'une part, la perception du changement climatique par les éleveurs et ses conséquences, d'autre part, leurs stratégies d'adaptation, 240 chefs de ménages ont été enquêtés, soit trente personnes dans chacun des huit villages sélectionnés. La méthodologie comprenait une étude exploratoire qui a permis la sélection des villages, une phase d'enquête approfondie avec I'administration des questionnaires auprès des pasteurs et agropasteurs, enfin des entretiens individuels et en groupe pour recueillir des informations complémentaires. Les résultats ont révélé que le changement climatique était perçu comme un phénomène qui affectait les pratiques d'élevage, très dépendantes de l'environnement. Les éleveurs ont noté une augmentation des températures (24\% des répondants), des épisodes de sécheresse plus longs (58\%) et une fréquence plus élevée des vents violents (18\%). Ces facteurs impactaient négativement l'activité pastorale de la commune et la rendaient très vulnérable. Pour y faire face, et notamment pour atténuer les effets de la sécheresse, les stratégies adoptées par les éleveurs incluaient le recours à la transhumance (25\%), l'achat d'aliments pour le bétail (22\%), le déstockage des animaux (15\%), et le stockage du fourrage $(15 \%)$. Les éleveurs utilisaient également un mélange de son et de glumes de mil ou de carton en cas de soudure difficile $(23 \%)$. Ces stratégies endogènes nécessitent d'être soutenues afin de les rendre plus performantes.
\end{abstract}

- Comment citer cet article : Abdou H., Karimou I.A., Harouna B.K., Zataou M.T., 2020. Perception of climate change among Sahelian pastoralists and strategies for adapting to environmental constraints: the case of the commune of Filingué in Niger. Rev. Elev. Med. Vet. Pays Trop., 73 (2): 81-90, doi : 10.19182/remvt.31873

\section{INTRODUCTION}

La notion de changement climatique est définie « comme étant des changements qui sont attribués directement ou indirectement à l'activité humaine altérant la composition de l'atmosphère mondiale et qui viennent s'ajouter à la variabilité naturelle du climat observée au cours

\footnotetext{
1. Université Boubacar BÂ de Tillabéri, Faculté des sciences agronomiques, Département des productions animales, BP 175, Tillabéri, Niger.

2. Université de Tahoua, Faculté des sciences agronomiques,

Département des productions animales, Tahoua, Niger.

* Auteur pour la correspondance

Tél. : +22796038018; +22780806518

Email : bassarou74@gmail.com
}

des périodes comparables » (GIEC, 2007). Par ailleurs, le changement climatique et ses conséquences sont désormais reconnus comme l'un des plus grands défis de la planète (GIEC, 2007 ; Ali et al., 2011 ; Bruno, 2012 ; Cheng et al., 2014). A l'échelle mondiale, on observe une élévation de la température moyenne et une nouvelle répartition des précipitations (Bergonzini, 2004). Ces modifications impactent la biodiversité et l'on estime que $15 \%$ à $37 \%$ des plantes et espèces animales terrestres pourraient disparaître d'ici 2050 (Clément, 2009 ; Bader et al., 2011). Ces modifications climatiques et écologiques contribuent, à côté d'autres facteurs anthropiques (augmentation des surfaces cultivées, déforestation, etc.), à l'accroissement de la vulnérabilité des populations et notamment celles pratiquant des activités agricoles comme l'élevage, qui sont très dépendantes de leur environnement.

$\mathrm{Au}$ Sahel, le changement climatique impacte très sévèrement les activités agricoles et pastorales. La région a été fortement marquée par des évènements climatiques extrêmes au cours des deux derniers 
siècles (GIEC, 2007 ; Mathieu et al., 2010 ; Hinsa, 2013 ; IRAM., 2013 ; Hiya et al., 2015). Au Burkina Faso, par exemple, Benoît (2008) identifie trois périodes remarquables depuis 1930 : la période humide (de 1930 à 1960), la période sèche dans les années 1970-1980, et la décennie 1990 à 2000, voire celle de 2000 à 2010, marquée par une légère reprise de la pluviométrie (INS-Niger, 2015 ; INS-Niger, 2018).

D'une manière générale, le changement climatique global se traduit, au niveau d'une localité, par plusieurs évolutions qui modifient les conditions de production (Degué, 2012). Ainsi, des décalages dans les calendriers climatiques, des variations dans la pluviométrie annuelle, et des périodes de sécheresse chroniques et répétitives ont été rapportés (Degué, 2012 ; Mathieu et al., 2010). De même, on observe l'apparition d'événements anormaux comme des cyclones et des températures anormalement élevées.

Pour relever ce défi majeur, il est apparu nécessaire de mettre en place des stratégies d'adaptation et de résilience à différents niveaux, de l'échelle de l'exploitation familiale à l'échelle globale en passant par les niveaux communautaire, national et régional. Le présent article discute des stratégies d'adaptation aux conditions environnementales changeantes mises en place localement par les pasteurs et agropasteurs de la commune urbaine de Filingué au Niger. En effet, il est remarqué dans cette commune une détérioration des ressources pastorales ainsi qu'une prolifération d'épizooties et des espèces non appétées rendant l'activité pastorale très vulnérable (Hinsa, 2013 ; IRAM, 2013 ; VSF, 2015). Face à cette situation, les pasteurs et agropasteurs de cette localité ont mis en place un certain nombre de stratégies d'adaptation afin de minimiser les impacts négatifs de ce phénomène sur l'élevage. Cheik Tidjane et al. (2012) rapportent que les populations du Sahel, qui sont celles qui subissent le plus les effets du changement climatique, disposent depuis des générations « d'un capital important d'expériences, de savoirs et de connaissances pour adapter [leurs activités] dont elles dépendent pour assurer leur survie. Il est donc nécessaire, pour une réponse durable aux changements climatiques, de mobiliser également l'expertise des communautés à la base ». Cette étude a eu pour objectif d'abord de comprendre la vulnérabilité des élevages de Filingué causée par les changements environnementaux, puis d'analyser les stratégies d'adaptation développées.

\section{MATERIEL ET METHODES}

\section{Zone d'étude}

L'étude a été conduite dans la commune urbaine de Filingué (14 $16^{\prime} \mathrm{N} ; 01^{\circ} 21^{\prime} \mathrm{E}$; figure 1). Située dans la partie nord-ouest du département de Filingué (région de Tillabéri), à environ 190 kilomètres de Niamey, cette commune compte cinquante-sept villages autour de Filingué. Elle s'étend sur environ 4800 kilomètres carrés.

Trois saisons distinctes caractérisent le département de Filingué : la saison froide ne dure plus que trois mois de nos jours (de décembre à février) et la saison chaude s'étend sur les autres mois à l'exception de la saison des pluies (de juillet à septembre). Les données météorologiques révèlent des conditions climatiques caractérisées par une forte température (INS-Niger, 2015 ; INS-Niger, 2018). Selon les observations de la Direction départementale de l'agriculture (DDA) du département en 2016, le régime thermique de la région a deux maxima, le premier en avril-mai avec une moyenne mensuelle de $41^{\circ} \mathrm{C}$, le second en octobre-novembre avec une moyenne de $38^{\circ} \mathrm{C}$. Un minimum de $18{ }^{\circ} \mathrm{C}$ est observé en décembre-février qui correspond à la saison la plus froide de l'année (DDA, 2016). Une variation thermique a été constatée au cours des deux dernières années. Les maxima, qui variaient autour de $41^{\circ} \mathrm{C}$ en 2016 selon la DDA/Filingué, oscillent de nos jours entre $45^{\circ} \mathrm{C}$ et $48^{\circ} \mathrm{C}$. Les relevés pluviométriques du département montrent que les pluies abondantes sont concentrées sur une période relativement courte, précisément au mois d'août, avec des poches de sécheresse très fréquentes. Au cours de la décennie 2006-2016, la pluviométrie était caractérisée par un volume à la fois insuffisant et une forte variabilité dans l'espace et dans le temps (INS-Niger, 2015). Le cumul annuel enregistré était de $456 \mathrm{~mm}$ en 25 jours en 2006 contre $545 \mathrm{~mm}$ en 30 jours en 2016 (DDA, 2016). Au cours de cette décennie, les plus faibles cumuls ont été enregistrés en 2007 et 2011, respectivement avec $319 \mathrm{~mm}$ (en 28 jours) et $271 \mathrm{~mm}$ (en 19 jours). Les variations interannuelles de la pluviométrie sont aussi très marquées (DDA, 2019). Pour les années 2017, 2018 et 2019, il a été enregistré respectivement $366 \mathrm{~mm}$ (en 33 jours), $318 \mathrm{~mm}$ (en 27 jours) et 408 en 34 jours.

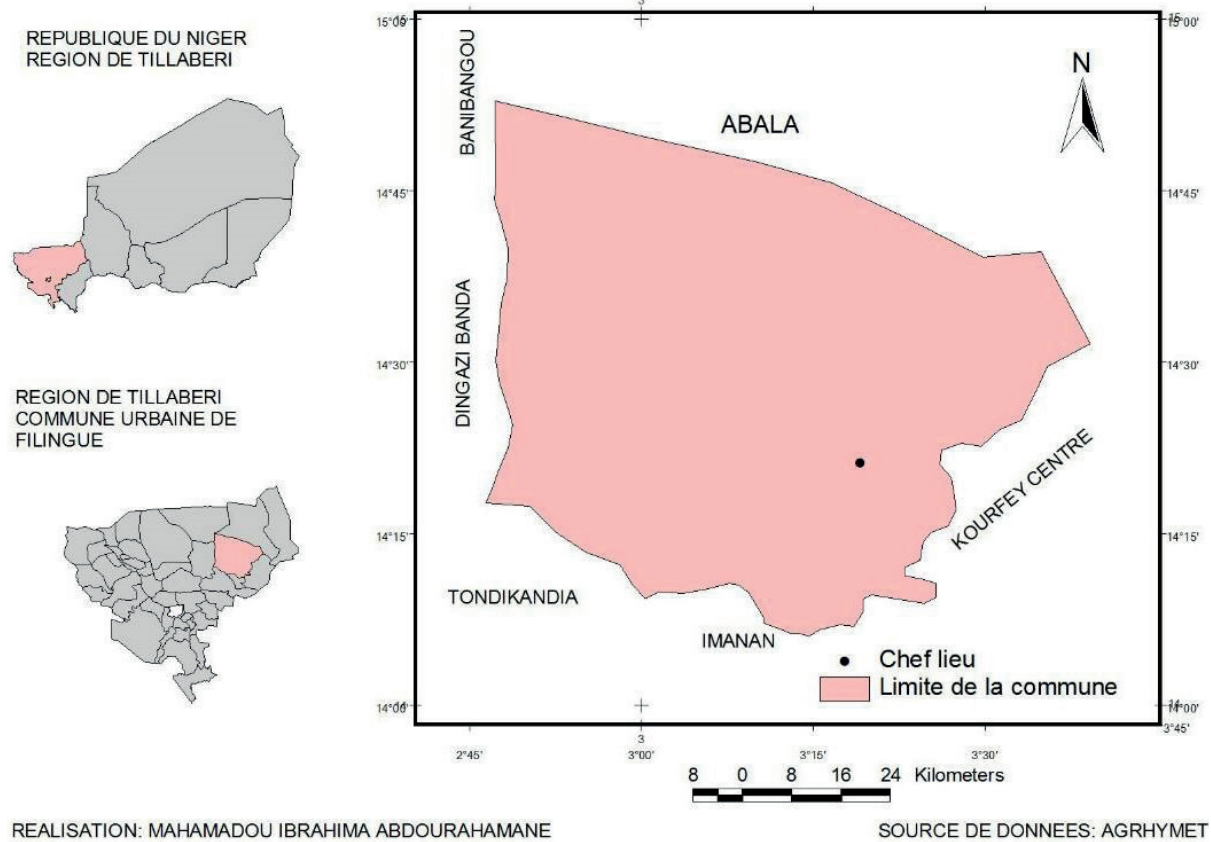

REPUBLIQUE DU NIGER REGION DE TILLABERI

REALISATION: MAHAMADOU IBRAHIMA ABDOURAHAMANE
Figure 1 : carte de la commune urbaine de Filingué au Niger (DDE, 2018). 
A Filingué, le couvert végétal est multiforme. Il se caractérise surtout par une certaine discontinuité très remarquée sur l'ensemble de la commune. Il est très lié à la nappe phréatique et à la nature du sol. Dans la zone des plateaux, la végétation est constituée par des formations de steppe arbustive à Combretaceae (notamment Combretum nigricans, Combretum micranthum, Guiera senegalensis). Le couvert herbacé est composé de Aristida mutabilis, A. pallida, Cenchrus biflorus, Zornia glochidiata et Eragrostis tremula (DDE/ SU/F, 2013). La végétation arborée des vallées est constituée par des parcs agroforestiers à Faidherbia albida, Hyphaene thebaica, Balanites aegyptiaca, Acacia nilotica et Acacia raddiana, et le couvert herbacé est composé de Aristida mutabilis, Aristida pallida, Cenchrus biflorus, Zornia glochidiata et Eragrostis tremula (DDE/SU/F, 2013). Dans la zone des plaines sablonneuses, la végétation est caractérisée par des peuplements à $F$. albida, A. raddiana, B. aegyptiaca, Ziziphus mauritiana et Acacia macrostachya (DDE/SU/F, 2013). Au Niger, il existe trois principaux systèmes d'élevage adaptés aux conditions agroécologiques des zones de production : le système pastoral, le système agropastoral, et le système urbain et périurbain.

\section{Système pastoral}

Comme son nom l'indique, ce type d'élevage se pratique de manière dominante dans la zone pastorale. Selon le code rural du Niger, il s'agit de la partie du pays située au nord de la limite de la zone des cultures, c'est-à-dire au nord de l'isohyète 350 millimètres. C'est une zone aride et subaride, où l'activité agricole non irriguée est peu développée. L'alimentation des animaux est basée sur les parcours naturels et sur la mobilité des troupeaux. La transhumance y est la forme de mobilité la plus courante et est pratiquée sur des distances plus ou moins longues. On trouve aussi des élevages pratiquant le nomadisme, c'est-à-dire avec des formes de mobilité moins régulières. Il convient de noter que cette mobilité peut être interne ou transfrontalière, sur de longues distances, à la recherche de meilleures conditions d'affouragement. Les principales destinations pour la transhumance transfrontalière sont le Nigeria, le Burkina Faso, le Bénin, le Tchad, le Mali, le Togo et le Cameroun (Rhissa, 2010). Les principaux acteurs de ce système d'élevage sont les Peulhs et les Touaregs qui effectuent des mouvements saisonniers dans les directions nord-sud et sud-nord (Marichatou et al., 2005). Dans ce déplacement, il est remarqué que le cheptel est constitué de races locales endurantes à la marche. Selon les acteurs, le coût des facteurs de production (alimentation, soins vétérinaires, eau...) est relativement faible.

\section{Système agropastoral}

Dans le système agropastoral, l'élevage est pratiqué généralement sur des parcours naturels avec un troupeau (bovin, ovin, caprin) de plus petite taille. Comparé au système pastoral, il repose sur la complémentarité entre l'agriculture et l'élevage et dépend donc de manière moins exclusive des parcours naturels. Les animaux bénéficient des résidus de récoltes et certains d'une complémentation basée sur l'achat de fourrages variés comme les sous-produits agroindustriels (Adamou, 2018). Cette complémentation est sélective et concerne généralement les animaux à l'embouche ou des femelles gravides ou en lactation. Les agropasteurs sont soit des agriculteurs sédentaires qui se sont diversifiés dans l'élevage de ruminants, soit des pasteurs qui se sont diversifiés dans l'agriculture (Geesing et Djibo, 2001) suite aux sécheresses des années antérieures (1970, 1974, 1984) ou suite à des changements de stratégies individuelles pour s'adapter aux contraintes locales.

\section{Système d'élevage urbain et périurbain}

A la différence des deux précédents systèmes, le mode d'élevage urbain et périurbain repose sur une intensification des pratiques d'élevage. Selon Vias et al. (2003), le troupeau de taille restreinte est soit homogène (une seule espèce) soit hétérogène (plusieurs espèces animales). Lélevage est parfois pratiqué en complément d'autres activités agricoles comme l'agriculture pluviale ou le maraîchage. Dans ce système, la pratique de la complémentation alimentaire occupe une grande place. Les coûts de production (notamment l'alimentation) sont ainsi plus élevés que dans les systèmes pastoraux ou agropastoraux. Certains élevages périurbains sont plus extensifs, comme les élevages peulhs localisés autour des grandes villes dans lesquels les animaux reçoivent le complément alimentaire le soir après le retour de pâturage sur les parcours naturels.

\section{Echantillonnage et collecte des données}

Pour la collecte des données, une enquête a été effectuée du 13 août au 19 septembre 2019 dans huit villages de la commune de Filingué, afin de recueillir les avis des chefs de ménages sur leurs perceptions du changement climatique, la vulnérabilité du secteur pastoral de cette commune face à ce changement ainsi que les stratégies développées par ces populations pour y faire face. Les villages retenus pour l'enquête ont été choisis sur la base de l'effectif de leur cheptel (gros et petit). La méthode utilisée pour sélectionner les personnes à interviewer a été celle du calcul des pas. Elle consiste à recenser et à numéroter tous les chefs de ménage pasteurs et agropasteurs du village, puis de les diviser par trente. Le quotient du rapport représente le pas. On tire ensuite au hasard un chiffre compris entre 1 et le chiffre obtenu suite à cette division. Le chiffre tiré représente le numéro de la première personne à enquêter, puis à chaque numéro trouvé on ajoute le chiffre obtenu suite à la division pour déterminer le prochain numéro. Ainsi, 240 personnes des deux sexes ont été interviewées (tableau I), à raison de 30 personnes par village.

\section{Tableau I}

Répartition des éleveurs selon le mode d'élevage dans huit villages de la commune urbaine de Filingué au Niger

\begin{tabular}{lrcc} 
Village & Pasteur & Agropasteur & Total \\
\hline Bada Bai & 1 & 29 & 30 \\
BakinToulou & 2 & 28 & 30 \\
Gaouna & 0 & 30 & 30 \\
Ouraparthé & 19 & 11 & 30 \\
Talcho & 1 & 29 & 30 \\
Tamagorzek & 0 & 30 & 30 \\
Tidiba & 9 & 21 & 30 \\
Yanta (quartier en ville) & 4 & 26 & 30 \\
Total & 36 & 204 & 240
\end{tabular}

Un questionnaire individuel d'enquête conduite en un seul passage a été soumis aux pasteurs et agropasteurs des huit villages de Filingué. Outre ce principal outil, une enquête par interview et observation directe a été conduite pour la prise en compte de certains aspects indispensables pour l'analyse, l'interprétation et les recommandations. Ainsi, des informations complémentaires ont été fournies par les encadreurs et les autres personnes ressources.

\section{Analyse des données}

Les données collectées ont été traitées avec les logiciels Excel et Spss. Excel a permis de créer les figures et le tableaux, et Spss de déterminer le nombre et la proportion des répondants pour chaque question. 


\section{RESULTATS}

\section{Description des systèmes pastoraux et agropastoraux}

Les 240 chefs de ménages interviewés étaient âgés de 39 à 85 ans avec $15 \%$ de pasteurs et $85 \%$ d'agropasteurs (tableau I). Trois ethnies étaient représentées : Haoussa (82\%), Peulh (17\%) et Touareg (1\%). Trois types d'élevage ont été identifiés : semi-intensif, intensif et ranching.

L'élevage semi-intensif était surtout pratiqué pour l'embouche des bovins et des petits ruminants. Les animaux étaient maintenus à l'étable où ils étaient nourris avec une ration comprenant des fourrages et des concentrés (son de céréales, graines et tourteau de coton, etc.). Pour ces ménages, l'embouche constituait une véritable source de revenus. Dans notre échantillon, 85 \% des ménages pratiquaient ce type d'élevage. Il s'agissait des agropasteurs, autrement dit des sédentaires, pour la plupart de l'ethnie Haoussa.

L'élevage extensif se caractérisait par la mobilité du bétail à l'intérieur du terroir. Les animaux étaient généralement sous la conduite d'un berger au pâturage. De retour la nuit au village, ils pouvaient recevoir un complément alimentaire (son, sel, etc.). Une partie des animaux était confiée aux bergers transhumants qui quittaient le terroir à la saison sèche (mars-juin) pour aller dans d'autres régions en traversant souvent les frontières du pays. Parmi les répondants, principalement constitués de nomades peulhs, $15 \%$ pratiquaient ce type d'élevage.

Le ranching reposait sur l'exploitation des pâturages naturels sur un terroir circonscrit, équipé de structures hydrauliques bien réparties en vue d'une exploitation des pâturages tout au long de l'année. Ce type d'élevage était pratiqué par le ranch de la commune de Filingué, celui de Toukounous, d'une superficie de 4474 hectares. Cette structure étatique créée en 1933 avait pour mission la multiplication et la valorisation des bovins de race Azawak.

\section{Perception paysanne du changement climatique Température}

Près du quart $(24 \%)$ des personnes interrogées ont affirmé que la température avait augmenté et que la saison chaude était plus longue qu'il y a 20 ou 25 ans.

\section{Pluviométrie}

La majorité $(58 \%)$ des répondants a affirmé que la pluviométrie avait diminué. Selon ces éleveurs, la diminution des précipitations s'est traduite par un démarrage tardif de la saison des pluies. Cette saison qui jadis débutait au $7^{\mathrm{e}}$ mois du calendrier agricole (mai) ne commençait à la période de l'enquête qu'au $9^{e}$ mois (juillet). Les éleveurs ont rapporté également un arrêt précoce de cette saison, le plus souvent dans le $11^{\mathrm{e}}$ mois (septembre) au lieu du $12^{\mathrm{e}}$ (octobre) auparavant. La saison des pluies n'a duré ainsi que trois mois dans les années ayant précédé l'enquête contre cinq mois dans le passé. Par ailleurs, les « poches » de sécheresse qui n’étaient observées par le passé qu'après les premières pluies se sont étalées ces dernières années jusqu'à la fin de la saison pluvieuse. De plus, les éleveurs ont affirmé que la pluviométrie était le plus souvent concentrée pendant une courte période (généralement en août) et provoquait des inondations.

\section{Vents forts et sableux}

Une partie significative (18\%) des personnes interrogées percevait le changement climatique à travers une augmentation des vents forts et sableux. Selon ces éleveurs, ces vents de sable recouvrent les fourrages secs avant que le nouveau couvert végétal n'atteigne un stade où il peut être consommé par les animaux.

\section{Perception des causes du changement climatique}

La majorité des ménages (52\%) a affirmé que le changement climatique était dû principalement à la déforestation. D'autres (37\%) pensaient qu'il était une sanction divine due au changement des comportements de la population. Ils ont remarqué une détérioration des liens de solidarité et d'entraide. Certains (11\%) n'avaient pas d'avis sur les causes du changement climatique.

\section{Perception paysanne des évolutions environnementales associées}

\section{Ressources pastorales}

La grande majorité (92\%) des chefs de ménages ont affirmé avoir constaté une dégradation de la disponibilité en fourrage, alors que pour $7 \%$ d'entre eux l'état du fourrage n'avait pas changé, et seulement $1 \%$ ont noté une augmentation de la disponibilité fourragère. Quatre causes de la dégradation des ressources fourragères ont été citées : l'insuffisance de pluie (45\% des réponses), l'extension des champs de cultures (32\%) qui réduit les aires de pâturage, le ramassage de la paille (21\%) qui réduit les réserves édaphiques en semences, et la prolifération d'espèces envahissantes (2\%) comme Sida cordifolia et Cassia mimosoides (figure 2). Ces légumineuses empêchent le bon développement de certaines graminées qui, selon les éleveurs, constituaient le meilleur fourrage.
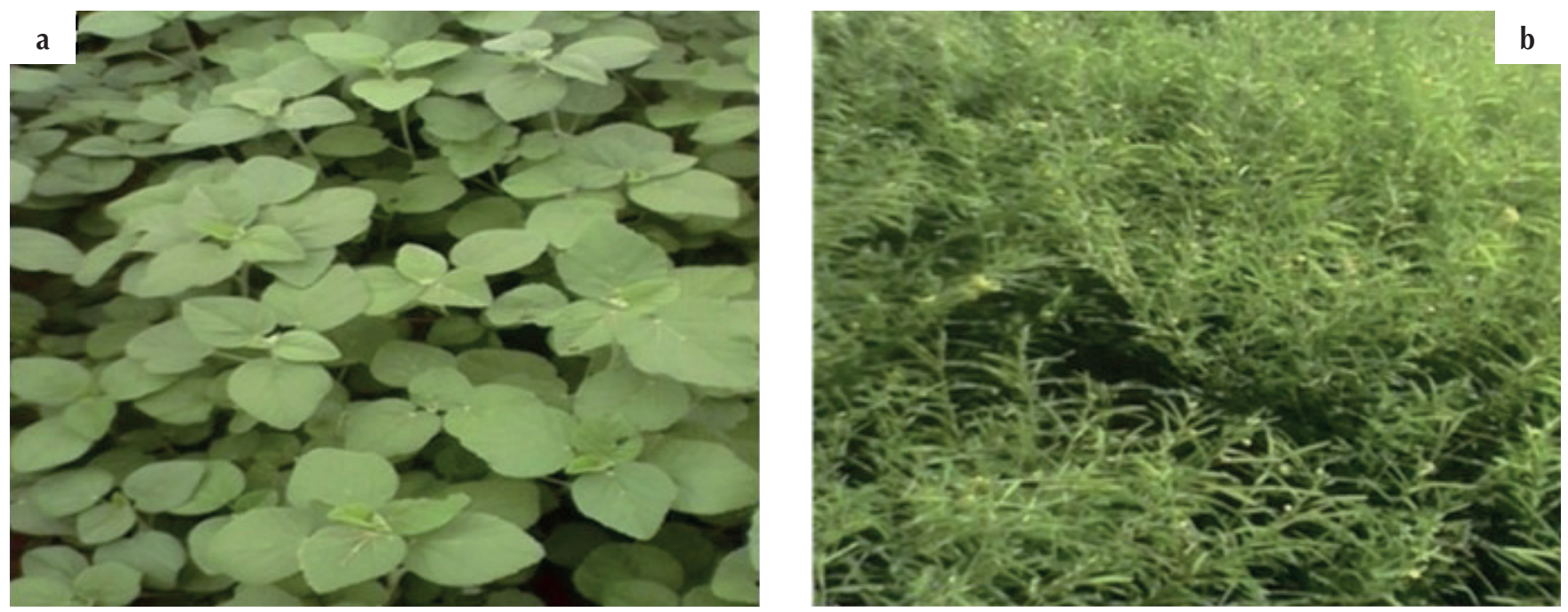

Figure 2 : espèces envahissantes ; (a) Sida cordifolia ; (b) Cassia mimosoides. 


\section{Paramètres zootechniques}

Ces évolutions ont impacté les performances des élevages (tableau II). Pour les petits ruminants, des changements pour certains paramètres zootechniques ont été rapportés, comme l'âge à la première mise bas qui, selon les enquêtes, a considérablement augmenté. Il était connu pour être à un an chez les petits ruminants et est passé à deux ans aujourd'hui. L'enquête a révélé que 82,4 \% des éleveurs partageaient cet avis contre $17,6 \%$ pour qui l'âge à la première mise base restait inchangé. Une augmentation de la durée de l'intervalle entre mises bas a été observée par $81,7 \%$ des éleveurs. Selon $86 \%$ des éleveurs, le changement climatique a aussi provoqué une augmentation de la fréquence des avortements, surtout chez les petits ruminants, à cause de l'augmentation de la température et des trajets parcourus à la recherche de pâturages. La même proportion d'éleveurs (86 \%) a rapporté une baisse de la vitesse de croissance des animaux à cause d'une insuffisance alimentaire.

Dans les huit villages, tous les pasteurs et agropasteurs ont constaté une diminution marquée de la productivité laitière des animaux. Pour confirmer cette affirmation, ils ont indiqué qu'une vache qui couvrait les besoins d'une famille en lait dans le passé pouvait à peine nourrir son veau de nos jours. La baisse des performances laitières des animaux est l'un des corollaires de l'insuffisance de fourrage (selon $13 \%$ des répondants) dont la dynamique est perçue comme un indicateur du changement climatique, mais surtout de la prolifération des parasites ( $87 \%$ des interviewés).

\section{Santé animale}

L'étude a révélé que $85 \%$ des éleveurs percevaient une détérioration de la santé animale liée aux épizooties, alors que $15 \%$ percevaient une amélioration de l'état sanitaire suite aux efforts des services vétérinaires et des organisations non gouvernementales qui interviennent en élevage. Le tableau III montre par ordre d'importance les cinq principales épizooties recensées dans la commune. Selon les éleveurs, ces épizooties avaient principalement pour origine la rencontre avec les animaux des éleveurs transhumants (65\% des avis). Les autres raisons évoquées étaient la prolifération de certains insectes vecteurs de maladies comme les moustiques (3\%), le déficit alimentaire qui affaiblit l'organisme de l'animal (4\%) et le manque d'hygiène des enclos (15\%) qui, après les pluies, dégagent de la chaleur ; $13 \%$ étaient sans avis. Certains éleveurs ont affirmé que la chaleur était à la base de l'apparition de la fièvre aphteuse.

\section{Changement sur l'entretien du bétail}

Pour les éleveurs, les changements environnementaux récents ont engendré des frais supplémentaires pour l'entretien du bétail. Ils ont déclaré (99\%) faire des dépenses énormes pour l'activité pastorale, soit pour l'achat d'aliment bétail pendant les périodes de sécheresse, soit pour la vaccination, soit pour les frais de gardiennage qui, d'après eux, avaient augmenté. Ces derniers étaient, d'après les enquêtes,

\section{Tableau II}

Avis des éleveurs sur les paramètres zootechniques dans la commune urbaine de Filingué au Niger

\begin{tabular}{lrrrr} 
Paramètre & $\begin{array}{c}\text { Age 1 } \\
\text { mise bas } \\
(\%)\end{array}$ & $\begin{array}{c}\text { Intervalle entre } \\
\text { mises bas } \\
(\%)\end{array}$ & $\begin{array}{c}\text { Avortements } \\
(\%)\end{array}$ & $\begin{array}{c}\text { Vitesse de } \\
\text { croissance } \\
(\%)\end{array}$ \\
\hline A augmenté & 82,4 & 81,7 & 86,2 & 1,2 \\
A diminué & 0 & 0,4 & 0,8 & 85,8 \\
Inchangé & 17,6 & 17,9 & 13 & 12,9
\end{tabular}

de 25 francs CFA par animal pour les petits ruminants et de 100 FCFA pour les bovins dans les années 2000, contre respectivement 50 FCFA et 150 FCFA pendant l'enquête. Le tableau IV récapitule les principaux facteurs environnementaux identifiés par les éleveurs comme impactant l'activité pastorale à Filingué.

\section{Stratégies d'adaptation mises en æuvre par les éleveurs}

\section{Stratégies contre la dégradation des ressources pastorales}

Face aux conséquences des évolutions environnementales sur l'activité pastorale, une panoplie de stratégies ont été adoptées par les pasteurs et agropasteurs pour freiner ce phénomène. Pour atténuer les effets de la sécheresse, les stratégies adoptées ont inclus le recours à la transhumance $(25 \%)$, l'achat d'aliments pour bétail (22\%), le déstockage des animaux (15\%), et le stockage du fourrage $(15 \%)$.

A l'approche de la saison des pluies ou après les premières pluies, période pendant laquelle le fourrage se fait rare (soudure), les agropasteurs ont utilisé une nouvelle technique de récupération des sous-produits alimentaires, à savoir les glumes de mil pour l'alimentation des animaux. Cette technique consistait à immerger les glumes de mil dans l'eau jusqu'à ce que le sable soit décanté au fond du récipient, puis à mélanger les glumes dans un autre récipient avec du son pour nourrir les animaux. En l'absence de glume de mil, certains éleveurs utilisaient des morceaux de cartons ramassés au marché. Cette stratégie qui assurait la valorisation des sous-produits, bien qu'étant nouvelle, était pratiquée par $23 \%$ des éleveurs.

Deux stratégies ont été adoptées par les éleveurs pour minimiser les dégâts causés par les inondations de plus en plus fréquentes : le recours à la transhumance (52\% des éleveurs) ou construire des enclos sur un terrain en hauteur (10\%). Plus d'un tiers (38\%) sont restés sans solution face aux inondations.

Pour lutter contre la propagation des feux de brousse, les éleveurs ont mis en place des bandes pare-feu ( $45 \%$ des réponses). En outre, $44 \%$ des éleveurs ont suivi des séances de sensibilisation de la population sur les dangers de l'usage du feu pour la gestion des pâturages. En effet, ces pratiques étaient à l'origine de nombreux feux de brousse.

\section{Stratégies contre les épizooties}

Pour lutter contre les épizooties, $61 \%$ des éleveurs ont eu recours au service de l'élevage pour le traitement et la prévention. Toutefois, $19 \%$ des éleveurs ont choisi la voie de la transhumance afin de préserver la santé de leur bétail. Selon ces éleveurs, certaines maladies avaient pour cause l'accumulation intense des déchets des animaux sur l'exploitation.

\section{Tableau III}

Epizooties principales recensées dans la commune urbaine de Filingué au Niger

\begin{tabular}{lc} 
Epizootie & $\%$ \\
\hline Clavelée & 29 \\
Fièvre aphteuse & 25 \\
Dermatose nodulaire & 20 \\
Peste bovine & 13 \\
Péripneumonie contagieuse bovine & 13
\end{tabular}




\section{Tableau IV}

Facteurs environnementaux impactant l'activité pastorale selon les éleveurs de la commune urbaine de Filingué au Niger

\begin{tabular}{|c|c|c|c|c|c|}
\hline \multirow[t]{2}{*}{ Facteur } & \multicolumn{3}{|c|}{ Impact } & \multirow{2}{*}{$\begin{array}{l}\text { Fréquence des } \\
\text { réponses (\% } \\
\text { des ménages } \\
\text { enquêtés) }\end{array}$} & \multirow{2}{*}{$\begin{array}{l}\text { Probabilité } \\
\text { de se } \\
\text { reproduire }\end{array}$} \\
\hline & Sur les resources pastorales & $\begin{array}{l}\text { Sur la santé } \\
\text { animale }\end{array}$ & Sur l'économie & & \\
\hline Sécheresse & $\begin{array}{l}\text { Diminution de la disponibilité des } \\
\text { ressources pastorales }\end{array}$ & $\begin{array}{l}\text { Augmentation } \\
\text { de la prévalence } \\
\text { d'épizooties }\end{array}$ & $\begin{array}{l}\text { Dépense supplémentaire } \\
\text { et perte de bétail }\end{array}$ & 100 & 2 ans \\
\hline Inondation & & & Perte de bétail & 49 & 7 ans \\
\hline Epizootie & & $\begin{array}{l}\text { Détérioration de } \\
\text { la santé animale }\end{array}$ & $\begin{array}{l}\text { Dépense supplémentaire } \\
\text { pour les soins }\end{array}$ & 100 & $\begin{array}{l}\text { Chaque } \\
\text { année }\end{array}$ \\
\hline $\begin{array}{l}\text { Feu de } \\
\text { brousse }\end{array}$ & Disponibilité du fourrage & $\begin{array}{l}\text { Trouble respiratoire } \\
\text { dû à la pollution } \\
\text { de l'air }\end{array}$ & $\begin{array}{l}\text { Dépense supplémentaire } \\
\text { pour l'achat de l'aliment } \\
\text { bétail }\end{array}$ & 3,3 & 14 ans \\
\hline Vol & & & $\begin{array}{l}\text { Diminution de l'économie } \\
\text { par perte de bétail }\end{array}$ & 76 & $\begin{array}{l}\text { Chaque } \\
\text { année }\end{array}$ \\
\hline $\begin{array}{l}\text { Vent fort } \\
\text { et sableux }\end{array}$ & $\begin{array}{l}\text { Dégradation des espaces pastoraux et des } \\
\text { points d'abreuvement } \\
\text { Diminution de la disponibilité de l'eau } \\
\text { d'abreuvement par l'ensablement des mares }\end{array}$ & $\begin{array}{l}\text { Diminution de } \\
\text { la qualité de l'air }\end{array}$ & Dépense pour l'entretien & 100 & $\begin{array}{l}\text { Chaque } \\
\text { année }\end{array}$ \\
\hline
\end{tabular}

\section{DISCUSSION}

\section{Perception paysanne du changement climatique}

A Filingué, le changement climatique est perçu par les paysans à travers plusieurs indicateurs au nombre desquels figurent les paramètres climatiques (la température, les pluies, les vents violents), et la production pastorale. Des résultats similaires ont été rapportés par Mathieu et al. (2010) sur la perception des paysans sur le changement climatique au Burkina Faso. Parmi les indicateurs paysans sur la perception du changement climatique, figuraient aussi un prolongement de la saison chaude, un démarrage tardif et un arrêt précoce de la saison pluvieuse, et une répartition inégale des précipitations dans l'espace et dans le temps.

A propos du changement climatique Dugué (2012) a écrit : «En effet, les activités humaines ont grandement contribué à l'augmentation de la concentration des gaz à effet de serre de telle sorte que l'équilibre écologique de la planète s'en est trouvé modifié. Ce bouleversement se traduit, aujourd'hui, par une amplification des dynamiques climatiques et une augmentation en fréquence et en intensité des événements climatiques hors normes : cyclones, sécheresses, fortes précipitations, vagues de chaleur. » De tels changements ont eu lieu dans le passé lointain, mais aujourd'hui ils n'ont jamais été aussi rapides.

L'action de l'homme sur la végétation se traduit par deux comportements majeurs : la déforestation et la pollution de l'atmosphère. Selon l'Organisation des Nations unies pour l'alimentation et l'agriculture (FAO, 1990), ce ne sont pas les vents et les pluies qui sont responsables de l'érosion accélérée, ni quelques défauts inhérents au sol, ce sont les humains qui en sont responsables. Les humains exercent leur action par méconnaissance ou par nécessité en favorisant souvent les phénomènes adverses qu'ils devraient combattre. La destruction des forêts et des pâturages à la suite d'une exploitation abusive, le défrichement des terres sur pentes trop accentuées, les techniques culturales inopportunes, la disparition de la matière organique des champs par l'exploitation des résidus des récoltes en sont des exemples

En quelques décennies, la population a beaucoup augmenté et l'exploitation des écosystèmes n'a ainsi cessé de s'accélérer. La croissance démographique s'est souvent accompagnée d'une extension des surfaces cultivées sur des terrains de moins en moins propices à la mise en culture. L'agressivité des conditions climatiques aggravera cette action destructrice sur l'environnement dans les pays sahéliens.

\section{Perception paysanne des évolutions environnementales associées}

A l'instar des autres régions du monde (Mathieu et al., 2010 ; Ali et al., 2011 ; Hinsa, 2013 ; IRAM., 2013 ; Hiya et al., 2015), la dynamique environnementale a impacté considérablement les ressources fourragères de Filingué. Les perceptions paysannes de l'impact du changement climatique sur ces ressources, recueillies lors de cette étude, laissent présager une dégradation de la quantité et de la qualité du fourrage. Lemaire et al. (2006) relient le mécanisme de cette dégradation à une insuffisance des pluies et à la prolifération d'espèces envahissantes comme Sida cordifolia qui empêche le développement des espèces appétées. De même, Amoukou (2009) écrit : «On a assisté à la disparition d'herbacées très appétées et à la prolifération d'autres de moindre valeur pastorale comme Sida cordifolia, mais bien adaptées aux nouvelles conditions écologiques ». Certaines pratiques anthropiques comme le ramassage de la paille, qui entraîne une diminution du stock édaphique en semences, et l'extension des champs de cultures sur les aires de pâturage, aggravent cette dégradation. Ces pratiques sont consécutives à la croissance démographique et à la baisse de la production agricole. Selon Vétérinaires sans frontières (VSF, 2015) « à cause de la croissance démographique les aires de pâturage, les couloirs de passage, les pistes de transhumance et les enclaves pastorales sont piétinés et se réduisent comme peau de chagrin ». 
Les éleveurs percevaient des changements importants en lien avec la dynamique des ressources pastorales. Comme pour toutes les ressources naturelles, la production des espèces fourragères au Sahel connaît, de façon générale, une baisse en saison sèche. Dans de telles conditions de sécheresse, le lien entre aridité et restriction de la nutrition azotée et minérale des espèces fourragères a été montré (Lemaire et al., 2006). En année de déficit hydrique important, on relève une baisse de plus de $50 \%$ de la production fourragère (Ruget et al., 2006). Dans certaines régions du monde, une collecte des informations sur les sécheresses en cours est mise en place afin d'anticiper des actions dans la conduite des systèmes fourragers. Le système Information et suivi objectif prairie (ISOP) proposé par Ruget et al. (2006) estime la variabilité interannuelle de production pour chaque région fourragère et l'évolution au cours de l'année de cette production, en valeur relative par rapport à la production «normale » de la même zone à la même date.

Les vents violents (tempêtes, cyclones, tornades et orages) figurent dans les indicateurs clés du changement climatique. A Filingué, une constante augmentation des vents forts et sableux a été observée par $52 \%$ des personnes interrogées. Ces vents violents peuvent affecter les hommes et les animaux directement ou indirectement (Bader et al., 2011 ; Cheng et al., 2014). Ils peuvent causer des blessures légères mais aussi des mortalités, notamment par des objets projetés, des chutes d'arbres ou des glissements de terrain (Gerber, 2012). Ils peuvent par ailleurs impacter l'économie par la destruction des abris aussi bien pour les humains que pour les animaux, ou le transport qui assure le ravitaillement. Ainsi, l'interruption des trafics (routier, ferroviaire, aérien) peut se traduire par des coûts, des pertes ou des perturbations d'activités importants, comme dans l'élevage (Gerber, 2012). Par ailleurs, les réseaux d'eau, téléphonique et électrique subissent à chaque tempête, à des degrés divers, des interruptions. Ceci peut entraîner une paralysie temporaire de la vie économique. Le milieu agricole paye régulièrement un lourd tribut aux tempêtes en raison des pertes de revenus résultant des dommages au bétail et aux cultures (Gastineau et Soden, 2009). Pour les atteintes portées à l'environnement (faune, flore, milieu terrestre et aquatique), on peut retenir la destruction de forêts par les vents et la pollution comme effet indirect. Par ailleurs, le Groupe intergouvernemental d'experts sur l'évolution du climat (GIEC, 2007) prévient que les impacts des changements climatiques se traduiront par une montée du niveau moyen de la mer, des pluies hivernales plus abondantes et des orages plus violents et plus fréquents.

D’une manière générale, l'élevage est une activité majeure qui procure des revenus dans les localités de Filingué. Malheureusement, les impacts des changements environnementaux ont entraîné une réduction de la surface de pâturage naturel, et il est établi que ceci est susceptible de continuer avec la diminution supplémentaire et l'irrégularité de la disponibilité de l'eau. Les inondations conjuguées aux conséquences de l'érosion hydrique et éolienne constituent un moteur important des pertes de pâturage. Ces phénomènes pourraient forcer le cheptel à migrer, ce qui conduirait à des conflits avec d'autres parties prenantes (agriculteurs, éleveurs et pêcheurs). L'impact de ces changements sur l'élevage est observé à trois niveaux : les paramètres zootechniques, la santé et les charges pour l'entretien du bétail (Hinsa, 2013).

L'étude a révélé une augmentation de l'âge à la première mise bas et des risques élevés d'avortement. En zootechnie expérimentale, les causes suspectées d'avortement sont la hausse de la température et l'allongement du temps de parcours des animaux à la recherche de pâturages (Lemaire et al., 2006 ; VSF, 2015). Concernant les paramètres de reproduction, les données expérimentales disponibles ne permettent pas de dégager le lien avec la perception des paysans sur la question car, de toute évidence, à l'origine, leurs protocoles n'étaient pas élaborés pour ce genre d'analyse. Ces études conduites sur les performances reproductives des animaux visaient, pour l'essentiel, la caractérisation des races dans des conditions agroclimatiques variées. Ainsi, Bartha (1963) rapporte un taux de fécondité de $85 \%$ à la station sahélienne expérimentale de Toukounous (département de Filingué). Boly et al. (2000) enregistrent un taux de $89 \%$ chez la même race en zone humide de la station de Loumbila au Burkina Faso, zone plus favorable que la station de Toukounous. Pour l'aptitude bouchère, l'âge au premier vêlage est un critère important pour l'appréciation de la précocité d'un animal ; sa valeur peut être influencée par des facteurs environnementaux comme l'alimentation. Achard et Chanono (1997) rapportent un intervalle entre vêlages de 36,5 \pm 4 mois chez le zébu Azawak à la station de Toukounous. Pour ce paramètre de reproduction, ils trouvent une différence entre les saisons de l'année au Niger. De plus, ils constatent que les femelles nées en début de saison des pluies ont un intervalle entre vêlages plus court que celles nées pendant le reste de l'année. A la station de Loumbila au Bukina Faso, Boly et al. (2000) rapportent un âge au premier vêlage de 32,6 mois chez la même race. Achard et Chanono (1995) enregistrent à Toukounous un intervalle entre vêlages de $14 \pm 2,9$ mois chez le zébu Azawak.

Les informations recueillies auprès des interviewés laissent présager un ralentissement marqué de la vitesse de croissance des animaux et une baisse de la production laitière notamment chez les bovins. Là encore, les études expérimentales assez poussées conduites dans la zone (Chanono, 2003) ne sont centrées que sur les variations saisonnières des performances. Les variations interannuelles de ces performances qui sont les plus indiquées pour élucider l'impact de la dynamique environnementale n'ont pas été abordées. Peu de travaux de recherche sont conduits sur la thématique dans cette zone et donc peu de données sont disponibles pour confronter les résultats de la présente étude à des performances mesurées. La mise en place d'un dispositif de suivi régulier de l'évolution des performances de reproduction et de production des animaux est nécessaire pour confirmer avec plus de précision les indicateurs de perception paysanne sur l'évolution de performances évoquées dans notre étude.

Selon les éleveurs de Filingué, la baisse de la production laitière est principalement due à l'insuffisance de fourrage mais elle pourrait aussi être due à une prolifération de certains arthropodes comme les tiques qui se fixent aux mamelles des vaches (Lemaire et al., 2006 ; GIEC, 2007 ; VSF, 2015). Le développement des insectes au cours de ces dernières années est favorisé par la hausse de la température (Hinsa, 2013 ; Cheng et al., 2014).

La santé est un indicateur pour le développement des organismes. Malheureusement, il est tributaire de l'état nutritionnel de l'organisme. Un déséquilibre éphémère ou chronique de l'état nutritionnel peut perturber la santé. Concernant la question de la santé animale face au changement climatique à Filingué, une augmentation de la fréquence des épizooties comme la fièvre aphteuse et la peste bovine a été notée. Une partie de la population interviewée attribuait l'intensification de ces épizooties à la rencontre des troupeaux autour des points d'abreuvement, au manque d'hygiène des enclos dû à l'accumulation des bouses, sources importantes de production de méthane (gaz à effet de serre) qui, après les pluies, dégageaient de la chaleur. Cette chaleur semble favoriser la fièvre aphteuse. Selon VSF (2015) l'intensification de ces épizooties est liée à une pollution de l'eau et des terres, et indirectement à l'accroissement des émissions de gaz à effet de serre.

\section{Stratégies adaptées}

La réduction des aires de pâturage, la raréfaction des points d'eau, l'apparition de nouvelles maladies animales, l'augmentation de la fréquence des sécheresses sont autant d'indicateurs qui attestent la 
vulnérabilité des ressources animales aux changements environnementaux et interpellent à la fois les éleveurs et les acteurs politiques sur l'urgence de prendre des mesures appropriées (N'djafa, 2009). Devant cette vulnérabilité chronique, des schémas ont été tracés comme les stratégies d'amélioration de l'alimentation du bétail à travers le monde (N’djafa, 2009 ; Cheikh et al., 2012 ; Degué, 2012).

\section{Stratégies contre la dégradation des ressources pastorales}

Afin de minimiser les effets des changements environnementaux sur l'activité pastorale, plusieurs stratégies ont été adoptées par les pasteurs et agropasteurs de Filingué comme la mobilité des animaux (transhumance). Selon Bruno (2012), la transhumance est « une stratégie de base pour s'adapter à la forte inégalité spatio-temporelle des ressources pastorales et hydrauliques. Elle permet également de compenser le faible taux de renouvellement de ces ressources ». Par définition, la transhumance est un déplacement globalement prévisible, saisonnier, des animaux vers certaines régions à la recherche de ressources pastorales (eau et fourrage). Cette pratique permettait aussi de protéger le bétail contre certaines épizooties. Selon les éleveurs, l'immobilité des animaux pourrait entraîner l'apparition de certaines épizooties suite à l'accumulation des déchets qui favorisent le développement de vecteurs (mouches, tiques et moustiques). L'importance de cette stratégie est aussi relevée dans une étude de l'IRAM (2013) qui montre que « la mobilité permet de gérer les aléas à savoir la disponibilité des pâturages et de l'eau, les situations de conflit ou d'insécurité, les inondations et les foyers de maladies en faisant varier les routes et les dates de transhumance ». Anderson et Monimart, (2009), et Hiya et al. (2015) rapportent que la transhumance constitue la meilleure stratégie de gestion des ressources naturelles et d'adaptation aux phénomènes majeurs et préoccupants du changement climatique pour une sécurisation de l'élevage au Sahel. A Falingué, les animaux se déplacent du sud vers le nord pendant la saison des pluies en relation avec la dynamique de la végétation. Le déplacement en sens inverse (nord-sud) a lieu après les récoltes et s'effectue en fonction de la pluviométrie annuelle enregistrée. Par conséquent, cet aller ou retour vers le sud peut être précoce ou retardé.

\section{- Déstockage}

Le déstockage est la stratégie utilisée par les éleveurs en vue de protéger une partie de leurs animaux, principalement les reproducteurs, en cas de déficit fourrager ou d'extrême soudure (Steve, 2009). Dans notre étude, cette pratique consistait à vendre une partie des troupeaux. La vente d'animaux contribuait à diminuer la pression sur les ressources naturelles et à donner aux éleveurs les moyens d'acheter des aliments pour bétail (son, paille) pour complémenter le reste du troupeau et assurer un minimum de suivi sanitaire des animaux.

\section{- Stockage du fourrage et achat d'aliment bétail}

Après les récoltes, pour prévenir la période de soudure, les agropasteurs stockent d'importantes quantités de fourrage constitué de résidus de cultures (tiges de mil et de sorgho, fanes de haricot) ou de bottes d'herbe (Steve, 2009 ; IRAM, 2013). Ce fourrage est donné aux animaux à la période de soudure afin de les aider à rester en bonne santé jusqu'à la saison des pluies. Malgré son avantage pour l'activité pastorale, cette stratégie présente des conséquences néfastes pour l'environnement car elle diminue la disponibilité des semences et engendre la disparition de certaines espèces herbacées (FAO, 1990). Il est à noter aussi que le ramassage de la paille met à nu le sol et l'expose à l'érosion hydrique et éolienne. Ce fourrage stocké ne couvre pas à lui seul les besoins alimentaires des animaux, d'où la nécessité d'acheter des compléments alimentaires (son, tourteau...).

Face à la dégradation quantitative et qualitative des ressources fourragères due au déficit de l'approvisionnement en eau qui limite la productivité des pâturages, les petits éleveurs tentent de s'adapter tant bien que mal à ces pénuries (Benoit, 2008 ; Mathieu et al., 2010 ; Hiya et al., 2015). Pour les petites exploitations familiales détentrices de bétail, des alternatives ont été développées pour combler le déficit alimentaire de leurs animaux et maintenir voire développer une production laitière source de revenus et de nourritures. Il s'agit du développement des cultures fourragères (niébé fourrager, maïs fourrager et sorgho fourrager), le fauchage, l'ensilage et la conservation de l'herbe pour l'alimentation du bétail en saison sèche (FAO/SFW, 2010).

\section{- Mélange du son et de la glume}

Ces stratégies d'adaptation, malgré leur importance, ne suffisaient pas pour répondre aux attentes des éleveurs. Pour cela, le gouvernement et les ONGs intervenant dans le domaine de l'élevage ont apporté leur soutien aux éleveurs en leur apprenant d'autres stratégies comme la confection des bandes pare-feu pour protéger le fourrage du feu de brousse, le traitement de la paille à l'urée, la distribution et la vente à prix modéré de l'aliment pour le bétail (son, tourteau...).

\section{Stratégies pour assurer le bien-être global des animaux}

Si ces communautés vivent les effets des changements environnementaux au quotidien, en marge de ces stratégies il serait opportun qu'elles soient mieux informées et outillées pour faire face à leurs conséquences. De fait, l'information et la sensibilisation ont une importance telle que les Nations unies les a considérées comme un axe stratégique majeur (Bader et al., 2011). L'information et la sensibilisation permettront ensemble une réflexion, des innovations locales de création et de renforcement des opportunités de collaboration autour des activités qui réduisent la vulnérabilité (Clément, 2009 ; Bader et al., 2011). Le résultat final pourrait être le renforcement des capacités d'adaptation aux changements et à la variabilité climatique (Gastineau et Soden, 2009).

\section{- CONCLUSION}

L'étude portant sur la perception paysanne de la dynamique environnementale menée dans la commune de Filingué a permis de comprendre que cette dynamique est une réalité pour les éleveurs de cette localité qui la perçoivent et modifient, en réponse, leur comportement. Le changement climatique est perçu par les paysans à travers la diminution de la pluviométrie et sa répartition inégale dans le temps, l'augmentation de la température, et des vents forts et sableux. Par ailleurs, l'enquête a révélé que ce bouleversement environnemental a entraîné des répercussions négatives sur la situation pastorale. En effet, la perturbation des systèmes productifs engendre la baisse de la production fourragère, la prolifération des épizooties et freine par conséquent le bon développement de l'activité pastorale. Les éleveurs rencontrent également d'énormes difficultés dans les déplacements des troupeaux et la transhumance qui constitue pourtant une stratégie vitale pour compenser les insuffisances en eau et en pâturages dans certaines localités.

Pour faire face aux nouvelles réalités agroécologiques, les paysans ont développé des stratégies endogènes leur permettant d'adapter leur activité. Mais ces stratégies ne répondent pas à toutes leurs attentes. A la lumière des résultats obtenus, il serait intéressant de poursuivre la réflexion sur les politiques et les projets à promouvoir pour atténuer les effets néfastes des changements environnementaux et encourager l'adaptation des activités pastorales à ces changements à Filingué et dans l'ensemble du Niger.

\section{Remerciements}

Cette étude a été entièrement financée par les auteurs. Toutefois, les auteurs remercient vivement l'ensemble du personnel de la Direction 
départementale de l'élevage de Filingué pour son soutien technique, moral et matériel pendant la conduite des enquêtes de terrain.

\section{Déclaration des contributions des auteurs}

HA a conçu, planifié et coordonné l'étude ; HA et MTZ ont recueilli les données ; HA a rédigé la première version du manuscrit ; HA et IAK ont effectué les analyses statistiques ; HA et IAK ont révisé toutes les versions du manuscrit ; BKH a révisé la première version du manuscrit

\section{Conflits d'intérêts}

L'étude a été réalisée sans aucun conflit d'intérêts.

\section{REFERENCES}

Achard F., Chanono M., 1997. Mortality and reproductive performances in Azawak zebus at the Station of Toukounous, Niger (1986-1992). Rev. Elev. Med. Vet. Pays trop., 50: 325-333, doi: 10.19182/remvt.9564

Adamou K.I., 2018. Performances de reproduction et de production laitière du taurin Kouri au Niger. Thèse Doct., Université Abdou Moumouni de Niamey, Niger, $135 \mathrm{p}$.

Ali C., Nadhem M., Hassen T., 2011. Impact du changement climatique sur la productivité des cultures céréalières dans la région de Béja (Tunisie). Afr. J. Agric. Res. Econ., 2 : 144-154

Amoukou A.I., 2009. Stratégies locales d'adaptation au changement climatique dans une zone rurale du bassin du Niger, Village nigérien face au changement climatique. Autorité du Bassin du Niger et la GTZ, Niamey, Niger, 100 p.

Anderson S., Monimart M., 2009. Recherche sur les stratégies d'adaptation des groupes pasteurs de la région de Diffa, Niger oriental. IIED, London, UK, $102 \mathrm{p}$

Bader J., Mesquita M.D.S., Hodges K.I., Keenlyside N., Østerhus S., Miles M., 2011. A review on Northern Hemisphere sea-ice, storminess and the North Atlantic oscillation: observations and projected changes. Atmos. Res., 101: 809-834

Bartha R., 1963. Elevage du zébu Azawak à la station de Toukounous (Niger) 1963-1967 (rapport). Station d'élevage de Toukounous, Niger, 55 p.

Benoit E., 2008. Les changements climatiques : vulnérabilité, impacts et adaptation dans le monde de la médecine traditionnelle au Burkina Faso. VertigO, 8 (1), doi : 10.4000/vertigo. 1467

Bergonzini J., 2004. Changements climatiques, désertification, diversité biologique et forêts. SILVA, Nogent-sur-Marne, France, 160 p.

Boly H., Some S.S., Kabre A., Sawadogo L., 2000. Reproduction et croissance du zébu Azawak en zone soudano-sahélienne (station de Loumbila au Burkina Faso). Ann. Univ. Ouagadougou, série B, 8 : 1-93

Bruno H., 2012. Changement climatique et conflits agropastoraux au Sahel. GRIP, Bruxelles, Belgique, 14 p.

Chanono M., 2003. Facteurs non génétiques influençant la production laitière du zébu Azawak à la station de Toukounous (Niger). Mém. DEA, Université de Liège, Faculté de médecine vétérinaire, Liège Belgique, 21 p.

Cheikh T.S., Mamadou F., Awa F.M., Bara G., 2012. Résilience et innovation locale face aux changements climatiques. Capitalisation des résultats du programme Fonds de soutien aux stratégies locales d'adaptation (FSSA). IED Afrique, Dakar, Sénégal, 43 p.

Cheng S.C., Lopes E., Fu C., Huang Z., 2014. Possible impacts of climate change on wind gusts under downscaled future climate conditions: update for Canada. J. Clim., 27: 1255-1270

Clément O., 2009. Perceptions, savoir locaux et stratégies d'adaptations aux changements climatiques des producteurs des communes d'Adjohoun et de Dangbo au Sud-est du Benin, 113 p.
Degué M.J., 2012. Caractérisation des stratégies d'adaptation au changement climatique en Agriculture paysanne, $50 \mathrm{p}$.

DDA, 2016. Rapports annuels des activités. Direction départementale de I'agriculture de Filingué (région de Tillabéri), Niger, 41 p.

DDA, 2019. Rapports annuels des activités. Direction départementale de I'agriculture de Filingué (région de Tillabéri), Niger, 40 p.

FAO, 1990. Conservation des sols et des eaux dans les zones semi-arides. FAO, Rome, Italie, 180 p. (Bull. pédologique ; 57)

FAO/SFW, 2010. Revue du secteur de I'élevage au Niger. FAO, Rome, Italie, $115 \mathrm{p}$.

Gastineau G., Soden B.J., 2009. Model projected changes of extreme wind events in response to global warning. Geophys Res. Lett., 36: L10810, doi: 10.1029/2009GL037500

Geesing D., Djido H., 2001. Profile fourrager du Niger. FAO, Rome, Italie

Gerber E.P., 2012. Stratospheric versus tropospheric control of the strength and structure of the Brewer-Dobson circulation. J. Atmos. Sci., 69: 28572877

GIEC, 2007. Bilan des changements climatiques : Rapport de synthèse. Contribution des groupes de travail au $4^{\mathrm{e}}$ rapport du groupe d'experts intergouvernemental sur l'évolution du climat, 103 p.

Hinsa R., 2013. Evaluation de la vulnérabilité et de I'adaptation du système de l'élevage des petits ruminants face au changement climatique. Cas du département de Say. Mém. Agrhymet, Niger, 100 p.

Hiya M., Andres L., Yamba B., Lebailly P., 2015. Mobilité pastorale au Sahel et en Afrique de l'Ouest. Université Abdou Moumouni, Niamey, Niger, $16 \mathrm{p}$.

INS, 2018. Annuaire statistique du Niger, 2013-2017. INS, Niger, 260 p.

INS-Niger, 2015. Annuaire statistique régional de Niamey 2010-2014. INS, Niger, $82 \mathrm{p}$.

IRAM, 2013. Systèmes d'élevage et changements climatiques au Tchad. IRAM, Tchad, $80 \mathrm{p}$.

Lemaire G., Duru M., Micold. Delaby L., Fiorelli J.L., Ruget F., 2006. Sensibilité à la sécheresse des systèmes fourragers et de l'élevage des herbivores. INRA, Versailles, France, 88-108

Marichatou H., Koré H., Motcho Henri K, Vias G., 2005. Synthèse bibliographique sur les filières laitières au Niger. Doc. travail $n^{\circ} 4$. Réseau de recherche et d'échanges sur les politiques laitières. ISRA-BAME, Dakar, Sénégal, $40 \mathrm{p}$.

Mathieu O., Youssouf D., Leopold S., 2010. Perceptions et stratégies d'adaptation aux changements des précipitations : cas des paysans du Burkina Faso. Sécheresse, $21: 87-96$

N'djafa H., 2009. Vulnérabilité, impacts et stratégies d'adaptation des populations locales à la variabilité et aux changements climatiques. SIFEE, Niamey, Niger, $16 \mathrm{p}$.

Rhissa Z., 2010. Revue du secteur de l'élevage, rapport provisoire. FAO, Rome, Italie, $115 \mathrm{p}$.

Ruget F., Moreau J.C., Cloppet E., Souverain F., 2006. Effect of climate change on grassland production for herbivorous livestock systems in France. Grassl. Sci., 15: 75-77

Steve A., 2009. Recherche sur les stratégies d'adaptation des groupes pasteurs de la région de Diffa, Niger oriental. IIED, London, UK, 102 p.

Vias G., Bonfoh B., Diarra A., Naferi A., Faye B., 2003. Les élevages laitiers bovins autour de la communauté urbaine de Niamey : caractéristiques, production, commercialisation et qualité du lait. In : Sémin. Lait sain pou le Sahel - production, approvisionnement, hygiène et qualité du lait et des produits laitiers au Sahel, Bamako, Mali, 24 fév.-3 mars 2003. Institut du Sahel, Bamako, Mali, 1-29

VSF., 2015. Elevage et changement climatique. Note de positionnement $n^{\circ} 2$ Synthèse. VSF, Bruxelles, Belgique, 4 p. 


\section{Summary}

Abdou H., Karimou I.A., Harouna B.K., Zataou M.T. Perception of climate change among Sahelian pastoralists and strategies for adapting to environmental constraints: the case of the commune of Filingué in Niger

The objective of the study was to understand the vulnerability of livestock farms to environmental changes in the urban commune of Filingué in Niger. In order to determine, on the one hand, the perception of climate change by livestock farmers and its consequences, and on the other hand, their adaptation strategies, 240 heads of households were surveyed, i.e. 30 people in each of the eight villages selected. The methodology consisted of an exploratory study to select the villages, an in-depth survey phase with the administration of questionnaires to pastoralists and agropastoralists, and individual and group interviews to gather additional information. The results revealed that climate change was perceived as a phenomenon that affected livestock husbandry practices, which are highly dependent on the environment. Livestock farmers noted an increase in temperature (24\% of respondents), longer droughts $(58 \%)$ and a higher frequency of high winds (18\%). These factors negatively affected the pastoral activity of the commune and made it very vulnerable. To cope with them, and in particular to mitigate the effects of droughts, the strategies adopted by the herders included the use of transhumance (25\%), feed purchase $(22 \%)$, destocking of animals $(15 \%)$, and fodder storage $(15 \%)$. Livestock farmers also used a mixture of bran with millet hulls or cardboard in case of hardship at the time preceding the harvest $(23 \%)$. These endogenous strategies need support to make them more effective.

Keywords: pastoralists, climate change adaptation, household surveys, vulnerability, resilience, Sahel, Niger

\section{Resumen}

Abdou H., Karimou I.A., Harouna B.K., Zataou M.T. Percepción de los criadores del cambio climático y estrategias de adaptación a los obstáculos ambientales: caso de la comuna de Filingué en Níger

El objetivo del estudio fue el de comprender la vulnerabilidad de las explotaciones de la comuna urbana de Filingué en Níger frente a los cambios climáticos ambientales. Con el fin de determinar, por una parte, la percepción del cambio climático de los criadores y sus consecuencias y, por otro lado, sus estrategias de adaptación, se encuestaron 240 jefes de familia, o sea treinta personas en cada uno de los ocho pueblos seleccionados. La metodología incluyó un estudio exploratorio que permitió la selección de los pueblos, una fase de encuesta profundizada con la administración de cuestionarios a los pastores y agro pastores, y en fin entrevistas individuales y en grupo para obtener informaciones complementarias. Los resultados revelaron que el cambio climático fue percibido como un fenómeno que afectaba las prácticas de cría, muy dependientes del ambiente. Los criadores notaron un aumento de las temperaturas (24\% de los respondientes), episodios de sequía más prolongados $(58 \%)$ y una frecuencia más elevada de vientos violentos $(18 \%)$. Estos factores impactaron negativamente la actividad pastoril de la comuna y la hicieron muy vulnerable. Para afrontarlos, y principalmente para atenuar los efectos de la sequía, las estrategias adoptadas por los criadores incluyeron el recurso a la trashumancia (25\%), la compra de alimentos para el ganado (22\%), la venta de animales $(15 \%)$ y el almacenamiento de forraje $(15 \%)$. Los criadores utilizaron también una mezcla de salvado con glumas de mijo o de cartones en caso de soldadura difícil (23\%). Estas estrategias endógenas deben ser mantenidas con el fin de hacerlas más rentables.

Palabras clave: pastoralistas, adaptación al cambio climático, encuestas de hogares, vulnerabilidad, resiliencia, Sahel, Níger 